\title{
Blow-up and global existence for solution of quasilinear viscoelastic wave equation with strong damping and source term
}

Jianghao Hao* (1) and Haiying Wei

\section{"Correspondence:} hjhao@sxu.edu.cn School of Mathematical Sciences, Shanxi University, Taiyuan, Shanxi 030006, China

\begin{abstract}
In this paper we consider a quasilinear viscoelastic wave equation with initial-boundary conditions, strong damping and source term. Under suitable assumptions on the initial data and the relaxation function, we establish a blow-up result of a solution for negative initial energy and some positive initial energy if the influence of the source term is greater than the dissipation. We show that the solution exists globally for any initial data if the influence of dissipation is greater than the source term.
\end{abstract}

Keywords: viscoelasticity wave equation; strong damping; blow-up; global existence

\section{Introduction}

In this work, we study the following quasilinear viscoelastic wave equation with initialboundary value conditions, strong damping and source term:

$$
\begin{cases}\left|u_{t}\right|^{\rho} u_{t t}-\Delta u+\int_{0}^{t} g(t-\tau) \Delta u(\tau) d \tau-\Delta u_{t}=|u|^{p-2} u, & \text { in } \Omega \times(0, \infty), \\ u(x, t)=0, & \text { on } \partial \Omega \times(0, \infty), \\ u(x, 0)=u_{0}(x), \quad u_{t}(x, 0)=u_{1}(x), & \text { in } \bar{\Omega},\end{cases}
$$

where $\Omega$ is a bounded domain of $R^{n}(n \geq 1)$ with smooth boundary $\partial \Omega, \rho>0$ and $p>2$ are constants. The relaxation function $g$ is a given function to be specified later.

As is well known, the wave equation with memory has been extensively studied. Berrimi and Messaoudi [1] considered the following initial-boundary value problem:

$$
\begin{cases}u_{t t}-\Delta u+\int_{0}^{t} g(t-\tau) \Delta u(\tau) d \tau=|u|^{\gamma} u, & \text { in } \Omega \times(0, \infty), \\ u(x, t)=0, & \text { on } \partial \Omega \times(0, \infty), \\ u(x, 0)=u_{0}(x), \quad u_{t}(x, 0)=u_{1}(x), & \text { in } \bar{\Omega},\end{cases}
$$

where $\Omega$ is a bounded domain of $R^{n}(n \geq 1)$ with smooth boundary $\partial \Omega, \gamma$ is a positive constant, and $g$ is a nonnegative and decreasing function. They obtained a local existence result and proved, for certain initial data and suitable conditions on $g$ and $\gamma$ (under weaker

(c) The Author(s) 2017. This article is distributed under the terms of the Creative Commons Attribution 4.0 International License (http://creativecommons.org/licenses/by/4.0/), which permits unrestricted use, distribution, and reproduction in any medium, provided you give appropriate credit to the original author(s) and the source, provide a link to the Creative Commons license, and indicate if changes were made. 
conditions than those in $[2,3]$ ), that the solution is global and decays uniformly (exponentially or polynomially depending on the decay rate of the relaxation function $g$ ) if the initial data is small enough. For further work on the existence and the decay of solutions, we refer the reader to [4-8]. Messaoudi [9] discussed the following initial-boundary value problem:

$$
\begin{cases}u_{t t}-\Delta u+\int_{0}^{t} g(t-\tau) \Delta u(\tau) d \tau+a\left|u_{t}\right|^{m-2} u_{t}=b|u|^{p-2} u, & \text { in } \Omega \times(0, \infty), \\ u(x, t)=0, & \text { on } \partial \Omega \times(0, \infty), \\ u(x, 0)=u_{0}(x), \quad u_{t}(x, 0)=u_{1}(x), & \text { in } \bar{\Omega},\end{cases}
$$

where $\Omega$ is a bounded domain of $R^{n}(n \geq 1)$ with smooth boundary $\partial \Omega, m \geq 1, p>2$, $a, b>0$ are constants and $g: R^{+} \rightarrow R^{+}$is a positive nonincreasing function. Under suitable conditions on $g$, he proved that solutions with negative initial energy blow up in finite time if $p>m$, and continue to exist if $p \leq m$. For the same problem, Messaoudi [10] extended this result to certain solutions with initial positive energy. A similar result was also obtained by Lu and Li [11], Guo and Lin [12].

Recently, Song and Zhong [13] studied a nonlinear viscoelastic problem with strong damping:

$$
\begin{cases}u_{t t}-\Delta u+\int_{0}^{t} g(t-\tau) \Delta u(\tau) d \tau-\Delta u_{t}=|u|^{p-2} u, & \text { in } \Omega \times(0, \infty), \\ u(x, t)=0, & \text { on } \partial \Omega \times(0, \infty), \\ u(x, 0)=u_{0}(x), \quad u_{t}(x, 0)=u_{1}(x), & \text { in } \bar{\Omega},\end{cases}
$$

where $\Omega$ is a bounded domain of $R^{n}(n \geq 1)$ with smooth boundary $\partial \Omega, 2<p<\frac{2 n-2}{n-2}$. They proved that solutions with positive initial energy blow up in finite time using the potential well method introduced by Payne and Sattinger [14]. Furthermore, Song and Xue [15] extended this result to arbitrarily high initial energy.

In the same direction Cavalcanti et al. [16] considered the following initial-boundary value problem:

$$
\begin{cases}\left|u_{t}\right|^{\rho} u_{t t}-\Delta u-\Delta u_{t t}+\int_{0}^{t} g(t-\tau) \Delta u(\tau) d \tau-\gamma \Delta u_{t}=0, & \text { in } \Omega \times(0, \infty), \\ u(x, t)=0, & \text { on } \partial \Omega \times(0, \infty), \\ u(x, 0)=u_{0}(x), \quad u_{t}(x, 0)=u_{1}(x), & \text { in } \bar{\Omega},\end{cases}
$$

where $\Omega$ is a bounded domain of $R^{n}(n \geq 1)$ with smooth boundary $\partial \Omega$ and $\rho>0$. They proved a global existence result for $\gamma \geq 0$ and an exponential decay result for $\gamma>0$. Cavalcanti et al. [17] studied problem (1.5) with $\rho \geq 0$ and $\gamma \geq 0$. The authors showed that the energy decays to zero uniformly with the rate that is determined from the solutions of the ODE quantifying the behavior of $g(t)$, and they improved many previous results. In the case of $\gamma=0$, Liu [18] discussed the following problem:

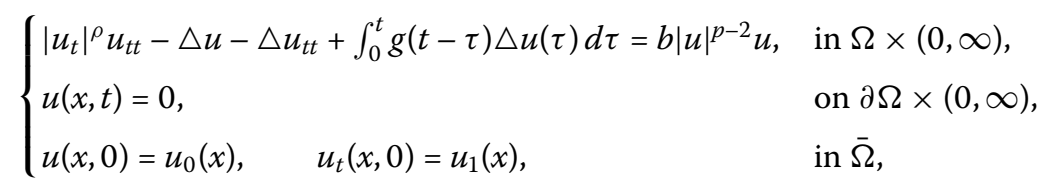


where $\Omega$ is a bounded domain of $R^{n}(n \geq 1)$ with smooth boundary $\partial \Omega$, and $\rho, b>0, p>2$ are constants. He obtained a general decay of the solution for certain class of relaxation functions and initial data in the stable set, and showed that the solution blows up in a larger class of initial positive energy. Furthermore, Song [19] studied the following problem:

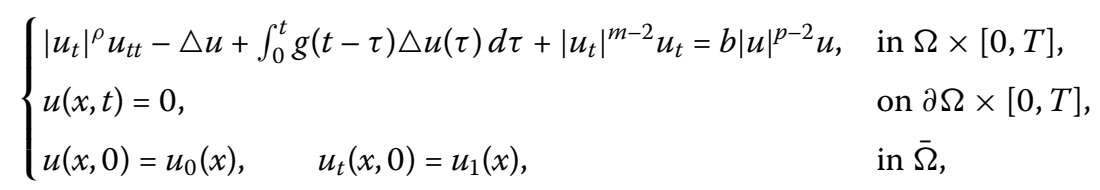

where $\Omega$ is a bounded domain of $R^{n}(n \geq 1)$ with smooth boundary $\partial \Omega, m>2, g: R^{+} \rightarrow R^{+}$ is a positive nonincreasing function. He proved the nonexistence of global solution of (1.7) with initial positive energy.

Motivated by the above pioneering work, we consider the problem (1.1). Under suitable assumptions on the initial data and the relaxation function $g$, we obtain a blow-up result for the solution with negative initial energy and some positive initial energy if $p>\rho+2$, and get a global existence result for any initial data if $p \leq \rho+2$ using the perturbed energy functional technique. This paper is organized as follows. In Section 2, we present some assumptions and preliminaries. Section 3 is devoted to the blow-up result. In Section 4, we obtain the global existence result.

\section{Preliminaries}

In this section, we shall give some notations and preliminaries used throughout this paper. Denote by $\|\cdot\|_{p}$ the usual norm in $L^{p}(\Omega)(p \geq 2)$. Let $B$ be the best embedding constant such that $\|\phi\|_{p} \leq B\|\nabla \phi\|_{2}, \phi \in H_{0}^{1}(\Omega)$. Besides, $C$ and $C_{i}\left(i \in N^{+}\right)$denote general positive constants, which may be different in different estimates.

Now, we make the following assumptions.

(G1) $g(t): R^{+} \rightarrow R^{+}$is a $C^{1}$ function satisfies

$$
\begin{aligned}
& g^{\prime}(s) \leq 0 \\
& 1-\int_{0}^{\infty} g(s) d s=l>0 .
\end{aligned}
$$

(G2) For the nonlinear term, we assume

$$
\left\{\begin{array}{l}
2<p<\infty, \quad \text { if } n=1,2 \quad \text { and } \quad 2<p \leq \frac{2(n-1)}{n-2}, \quad \text { if } n \geq 3 \\
0<\rho<\infty, \quad \text { if } n=1,2 \text { and } \quad 2<\rho \leq \frac{2}{n-2}, \quad \text { if } n \geq 3
\end{array}\right.
$$

We first state, without a proof, a local existence theorem which can be established by the Faedo-Galerkin method. The interested reader can refer to Cavalcanti et al. [5] for details.

Theorem 2.1 Assume (G1) and (G2) hold and $\left(u_{0}, u_{1}\right) \in H_{0}^{1}(\Omega) \times L^{2}(\Omega)$ is given. Then problem (1.1) has a unique local solution

$$
u \in C\left([0, T] ; H_{0}^{1}(\Omega)\right), \quad u_{t} \in C\left([0, T] ; H_{0}^{1}(\Omega)\right) .
$$


Lemma 2.2 Assume (G1) and (G2) hold. Let $u(t)$ be a solution of (1.1). Then $E(t)$ is nonincreasing. Moreover, for $t>0$, the following inequality holds:

$$
E^{\prime}(t)=-\left\|\nabla u_{t}(t)\right\|_{2}^{2}+\frac{1}{2}\left(g^{\prime} \circ \nabla u\right)(t)-\frac{1}{2} g(t)\|\nabla u(t)\|_{2}^{2} \leq 0,
$$

where

$$
E(t)=\frac{1}{\rho+2}\left\|u_{t}\right\|_{\rho+2}^{\rho+2}+\frac{1}{2}\left(1-\int_{0}^{t} g(s) d s\right)\|\nabla u(t)\|_{2}^{2}+\frac{1}{2}(g \circ \nabla u)(t)-\frac{1}{p}\|u(t)\|_{p}^{p}
$$

and

$$
(g \circ v)=\int_{0}^{t} g(t-\tau)\|v(t)-v(\tau)\|_{2}^{2} d \tau
$$

Proof Multiplying the first equation of (1.1) by $u_{t}$, integrating over $\Omega$, we obtain (2.5).

Lemma 2.3 ([18], Lemma 2.2) Assume (G1) and (G2) hold. Let $u(t)$ be a solution of (1.1). Assume further that

$$
E(0)<E_{1}=\left(\frac{1}{2}-\frac{1}{p}\right) B_{1}^{-\frac{2 p}{p-2}}
$$

and

$$
\left\|\nabla u_{0}\right\|_{2} \geq B_{1}^{-\frac{p}{p-2}}
$$

where $B_{1}=\frac{B}{\sqrt{l}}$. Then there exists a constant $\beta>B_{1}^{-\frac{p}{p-2}}$ such that for $t>0$

$$
\left(1-\int_{0}^{t} g(s) d s\right)\|\nabla u(t)\|_{2}^{2} \geq \beta^{2}
$$

and

$$
\|u\|_{p} \geq B_{1} \beta
$$

Lemma 2.4 For $2 \leq p \leq \rho+2$, we have

$$
\left\|u_{t}\right\|_{p}^{p} \leq\left\|u_{t}\right\|_{p}^{2}+\left\|u_{t}\right\|_{\rho+2}^{\rho+2}
$$

Proof If $\left\|u_{t}\right\|_{p}<1$, then we get $\left\|u_{t}\right\|_{p}^{p} \leq\left\|u_{t}\right\|_{p}^{2}$. If $\left\|u_{t}\right\|_{p} \geq 1$, then have

$$
\left\|u_{t}\right\|_{p}^{p} \leq C\left\|u_{t}\right\|_{p}^{\rho+2} \leq C\left\|u_{t}\right\|_{\rho+2}^{\rho+2}
$$

Together with the two cases, we obtain (2.8). 


\section{Blow-up result}

In this section we state and prove the blow-up result.

Theorem 3.1 Assume that (G1) and (G2) hold and

$$
\int_{0}^{\infty} g(s) d s<\frac{p / 2-1}{p / 2-1+1 / 2 p}
$$

Assume further that $p>\rho+2$, and $\left(u_{0}, u_{1}\right) \in H_{0}^{1}(\Omega) \times L^{2}(\Omega)$ is given. Then the solution $u(t)$ of problem (1.1) blows up in finite time, i.e. there exists $T_{0}<+\infty$ such that

$$
\lim _{t \rightarrow T_{0}^{-}}\left(\left\|u_{t}\right\|_{\rho+2}^{\rho+2}+\|\nabla u\|_{2}^{2}+\|u\|_{p}^{p}\right)=\infty
$$

if

$$
E(0)<\left(1-\frac{1}{p(p-2)} \frac{1-l}{l}\right)\left(\frac{1}{2}-\frac{1}{p}\right) B_{1}^{-\frac{2 p}{p-2}}
$$

and

$$
\left\|\nabla u_{0}\right\|_{2}>B_{1}^{-\frac{p}{p-2}}
$$

Proof Assume that there exists some positive constant $C$ such that for $t>0$ the solution $u(t)$ of (1.1) satisfies

$$
\left\|u_{t}\right\|_{\rho+2}^{\rho+2}+\|\nabla u\|_{2}^{2}+\|u\|_{p}^{p} \leq C .
$$

We set

$$
H(t)=E_{2}-E(t),
$$

where the constant $E_{2} \in\left(E(0), E_{1}\right)$ shall be chosen later. By Lemma 2.2,

$$
H^{\prime}(t)=-E^{\prime}(t) \geq 0 .
$$

Then, for $0 \leq s \leq t$, we have

$$
0<H(0) \leq H(s) \leq H(t)=E_{2}-E(t) .
$$

From (2.6), we have

$$
\begin{aligned}
H(t) & =E_{2}-E(t) \\
& =E_{2}-\frac{1}{\rho+2}\left\|u_{t}\right\|_{\rho+2}^{\rho+2}-\frac{1}{2}\left(1-\int_{0}^{t} g(s) d s\right)\|\nabla u(t)\|_{2}^{2}-\frac{1}{2}(g \circ \nabla u)(t)+\frac{1}{p}\|u(t)\|_{p}^{p} \\
& \leq E_{2}-\frac{1}{2}\left(1-\int_{0}^{t} g(s) d s\right)\|\nabla u(t)\|_{2}^{2}+\frac{1}{p}\|u(t)\|_{p}^{p}
\end{aligned}
$$




$$
\begin{aligned}
& \leq E_{1}-\frac{1}{2}\left(1-\int_{0}^{t} g(s) d s\right)\|\nabla u(t)\|_{2}^{2}+\frac{1}{p}\|u(t)\|_{p}^{p} \\
& \leq E_{1}-\frac{1}{2} B_{1}^{-\frac{2 p}{p-2}}+\frac{1}{p}\|u(t)\|_{p}^{p} \\
& \leq\left(\frac{1}{2}-\frac{1}{p}\right) B_{1}^{-\frac{2 p}{p-2}}-\frac{1}{2} B_{1}^{-\frac{2 p}{p-2}}+\frac{1}{p}\|u(t)\|_{p}^{p} \\
& \leq \frac{1}{p}\|u(t)\|_{p}^{p}
\end{aligned}
$$

Define

$$
L(t)=H^{1-\sigma}(t)+\frac{\varepsilon}{\rho+1} \int_{\Omega}\left|u_{t}\right|^{\rho} u_{t} u d x+\frac{\varepsilon}{2} \int_{\Omega}|\nabla u|^{2} d x,
$$

where the constant $\varepsilon>0$ shall be chosen later and the constant $\sigma$ satisfies

$$
0<\sigma<\frac{1}{\rho+2}-\frac{1}{p}
$$

Taking a derivative of (3.9) and using Lemma 2.2, we have

$$
\begin{aligned}
L^{\prime}(t)= & (1-\sigma) H^{-\sigma}(t)\left(\left\|\nabla u_{t}(t)\right\|_{2}^{2}-\frac{1}{2}\left(g^{\prime} \circ \nabla u\right)(t)+\frac{1}{2} g(t)\|\nabla u(t)\|_{2}^{2}\right) \\
& +\frac{\varepsilon}{\rho+1}\left\|u_{t}\right\|_{\rho+2}^{\rho+2}+\varepsilon \int_{\Omega}\left|u_{t}\right|^{\rho} u_{t t} u d x+\varepsilon \int_{\Omega} \nabla u_{t} \nabla u d x \\
\geq & (1-\sigma) H^{-\sigma}(t)\left\|\nabla u_{t}(t)\right\|_{2}^{2}+\frac{\varepsilon}{\rho+1}\|u\|_{\rho+2}^{\rho+2}-\varepsilon\|\nabla u\|_{2}^{2}+\varepsilon\|u\|_{p}^{p} \\
& -\varepsilon \int_{\Omega} \int_{0}^{t} g(t-\tau) \triangle u(\tau) d \tau u(t) d x .
\end{aligned}
$$

For the last term on the right side of (3.11), using the Green formula, we get

$$
\begin{aligned}
& -\int_{\Omega} \int_{0}^{t} g(t-\tau) \Delta u(\tau) d \tau u(t) d x \\
& =\int_{0}^{t} g(t-\tau) \int_{\Omega} \nabla u(\tau) \nabla u(t) d x d \tau \\
& =\int_{0}^{t} g(t-\tau) \int_{\Omega} \nabla u(t) \nabla(u(\tau)-u(t)) d x d \tau+\int_{0}^{t} g(t-\tau)\|\nabla u(t)\|_{2}^{2} d \tau \\
& =\int_{0}^{t} g(t-\tau) \int_{\Omega} \nabla u(t)(\nabla u(\tau)-\nabla u(t)) d x d \tau+\int_{0}^{t} g(\tau) d \tau\|\nabla u(t)\|_{2}^{2} .
\end{aligned}
$$

Substituting (3.12) into (3.11), we obtain

$$
\begin{aligned}
L^{\prime}(t) \geq & (1-\sigma) H^{-\sigma}(t)\left\|\nabla u_{t}(t)\right\|_{2}^{2}+\frac{\varepsilon}{\rho+1}\left\|u_{t}\right\|_{\rho+2}^{\rho+2}+\varepsilon\|u\|_{p}^{p} \\
& +\varepsilon \int_{0}^{t} g(t-\tau) \int_{\Omega} \nabla u(t)(\nabla u(\tau)-\nabla u(t)) d x d \tau \\
& -\varepsilon\left(1-\int_{0}^{t} g(\tau) d \tau\right)\|\nabla u(t)\|_{2}^{2} .
\end{aligned}
$$


Using the Cauchy inequality, for $0<\varepsilon_{1}<1$ we have

$$
\begin{gathered}
\int_{0}^{t} g(t-\tau) \int_{\Omega} \nabla u(t)(\nabla u(\tau)-\nabla u(t)) d x d \tau \\
\geq-\frac{p\left(1-\varepsilon_{1}\right)}{2} \int_{0}^{t} g(t-\tau)\|\nabla u(\tau)-\nabla u(t)\|_{2}^{2} d \tau \\
\quad-\frac{1}{\left(1-\varepsilon_{1}\right) 2 p} \int_{0}^{t} g(\tau) d \tau\|\nabla u(t)\|_{2}^{2} .
\end{gathered}
$$

By (3.14), we know

$$
\begin{aligned}
& L^{\prime}(t) \geq(1-\sigma) H^{-\sigma}(t)\left\|\nabla u_{t}(t)\right\|_{2}^{2}+\frac{\varepsilon}{\rho+1}\left\|u_{t}\right\|_{\rho+2}^{\rho+2}+\varepsilon\|u\|_{p}^{p} \\
& -\varepsilon\left(1-\int_{0}^{t} g(\tau) d \tau\right)\|\nabla u(t)\|_{2}^{2} \\
& -\varepsilon\left(\frac{p\left(1-\varepsilon_{1}\right)}{2} \int_{0}^{t} g(t-\tau)\|\nabla u(\tau)-\nabla u(t)\|_{2}^{2} d \tau\right. \\
& \left.+\frac{1}{\left(1-\varepsilon_{1}\right) 2 p} \int_{0}^{t} g(\tau) d \tau\|\nabla u(t)\|_{2}^{2}\right) \\
& \geq(1-\sigma) H^{-\sigma}(t)\left\|\nabla u_{t}(t)\right\|_{2}^{2}+\varepsilon\left(\frac{1}{\rho+1}+\frac{p\left(1-\varepsilon_{1}\right)}{\rho+2}\right)\left\|u_{t}\right\|_{\rho+2}^{\rho+2}+\varepsilon\left(1-\varepsilon_{1}\right) p\left(E_{2}-E(t)\right) \\
& +\varepsilon\left(\left(\frac{p\left(1-\varepsilon_{1}\right)}{2}-1\right)\left(1-\int_{0}^{t} g(\tau) d \tau\right)\|\nabla u(t)\|_{2}^{2}\right. \\
& \left.-\frac{1}{\left(1-\varepsilon_{1}\right) 2 p} \int_{0}^{t} g(\tau) d \tau\|\nabla u(t)\|_{2}^{2}\right) \\
& -\varepsilon\left(1-\varepsilon_{1}\right) p E_{2}+\varepsilon \varepsilon_{1}\|u\|_{p}^{p} \\
& =(1-\sigma) H^{-\sigma}(t)\left\|\nabla u_{t}(t)\right\|_{2}^{2}+\varepsilon\left(\frac{1}{\rho+1}+\frac{p\left(1-\varepsilon_{1}\right)}{\rho+2}\right)\left\|u_{t}\right\|_{\rho+2}^{\rho+2}+\varepsilon\left(1-\varepsilon_{1}\right) p H(t) \\
& +\varepsilon\left(\left(\frac{p\left(1-\varepsilon_{1}\right)}{2}-1\right)\left(1-\int_{0}^{t} g(\tau) d \tau\right)\|\nabla u(t)\|_{2}^{2}\right. \\
& \left.-\frac{1}{\left(1-\varepsilon_{1}\right) 2 p} \int_{0}^{t} g(\tau) d \tau\|\nabla u(t)\|_{2}^{2}\right) \\
& -\varepsilon\left(1-\varepsilon_{1}\right) p E_{2}+\varepsilon \varepsilon_{1}\|u\|_{p}^{p} \text {. }
\end{aligned}
$$

For the fourth term on the right side of (3.15), by (2.2) and Lemma 2.3, we obtain

$$
\begin{aligned}
& \left(\frac{p\left(1-\varepsilon_{1}\right)}{2}-1\right)\left(1-\int_{0}^{t} g(\tau) d \tau\right)\|\nabla u(t)\|_{2}^{2}-\frac{1}{\left(1-\varepsilon_{1}\right) 2 p} \int_{0}^{t} g(\tau) d \tau\|\nabla u(t)\|_{2}^{2} \\
& =\frac{\left(\frac{p\left(1-\varepsilon_{1}\right)}{2}-1\right)\left(1-\int_{0}^{t} g(\tau) d \tau\right)-\frac{1}{\left(1-\varepsilon_{1}\right) 2 p} \int_{0}^{t} g(\tau) d \tau}{1-\int_{0}^{t} g(\tau) d \tau}\left(1-\int_{0}^{t} g(\tau) d \tau\right)\|\nabla u(t)\|_{2}^{2} \\
& \geq \frac{\left(\frac{p\left(1-\varepsilon_{1}\right)}{2}-1\right) l-\frac{1}{\left(1-\varepsilon_{1}\right) 2 p}(1-l)}{1-\int_{0}^{t} g(\tau) d \tau} \beta^{2} .
\end{aligned}
$$


Then, by (3.15) and (3.16), we have

$$
\begin{aligned}
L^{\prime}(t) \geq & (1-\sigma) H^{-\sigma}(t)\left\|\nabla u_{t}(t)\right\|_{2}^{2}+\varepsilon\left(\frac{1}{\rho+1}+\frac{p\left(1-\varepsilon_{1}\right)}{\rho+2}\right)\left\|u_{t}\right\|_{\rho+2}^{\rho+2}+\varepsilon\left(1-\varepsilon_{1}\right) p H(t) \\
& +\frac{\left(\frac{p\left(1-\varepsilon_{1}\right)}{2}-1\right) l-\frac{1}{\left(1-\varepsilon_{1}\right) 2 p}(1-l)}{1-\int_{0}^{t} g(\tau) d \tau} \varepsilon \beta^{2}-\varepsilon\left(1-\varepsilon_{1}\right) p E_{2}+\varepsilon \varepsilon_{1}\|u\|_{p}^{p} .
\end{aligned}
$$

Since

$$
\int_{0}^{\infty} g(s) d s<\frac{p / 2-1}{p / 2-1+1 / 2 p}
$$

we have

$$
\left(\frac{p}{2}-1\right)\left(1-\int_{0}^{\infty} g(\tau) d \tau\right)-\frac{1}{2 p} \int_{0}^{\infty} g(\tau) d \tau>0 .
$$

It is easy to see that there exists $\varepsilon_{1}^{*}>0$, such that, for $0<\varepsilon_{1}<\varepsilon_{1}^{*}$,

$$
\frac{\left(\frac{p\left(1-\varepsilon_{1}\right)}{2}-1\right) l-\frac{1}{\left(1-\varepsilon_{1}\right) 2 p}(1-l)}{1-\int_{0}^{t} g(\tau) d \tau} \beta^{2}>\frac{\left(\frac{p\left(1-\varepsilon_{1}\right)}{2}-1\right) l-\frac{1}{\left(1-\varepsilon_{1}\right) 2 p}(1-l)}{1-\int_{0}^{t} g(\tau) d \tau} B_{1}^{-\frac{2 p}{p-2}} .
$$

Since

$$
E(0)<\left(\frac{1}{2}-\frac{1}{p}\right)\left(1-\frac{1}{p(p-2)} \frac{1-l}{l}\right) B_{1}^{-\frac{2 p}{p-2}}=\frac{\left(\frac{p}{2}-1\right) l-\frac{1}{2 p}(1-l)}{p l} B_{1}^{-\frac{2 p}{p-2}}
$$

we may choose $0<\varepsilon_{1}<1$ sufficiently small, and $E_{2} \in\left(E(0), E_{1}\right)$ sufficiently near $\mathrm{E}(0)$, such that

$$
\frac{\left(\frac{p\left(1-\varepsilon_{1}\right)}{2}-1\right) l-\frac{1}{\left(1-\varepsilon_{1}\right) 2 p}(1-l)}{1-\int_{0}^{t} g(\tau) d \tau} B_{1}^{-\frac{2 p}{p-2}}-p\left(1-\varepsilon_{1}\right) E_{2} \geq 0 .
$$

Then, for $t>0$, by (3.17) and (3.19), we obtain

$$
\begin{aligned}
L^{\prime}(t) \geq & (1-\sigma) H^{-\sigma}(t)\left\|\nabla u_{t}(t)\right\|_{2}^{2}+\varepsilon\left(\frac{1}{\rho+1}+\frac{p\left(1-\varepsilon_{1}\right)}{\rho+2}\right)\left\|u_{t}\right\|_{\rho+2}^{\rho+2} \\
& +\varepsilon\left(1-\varepsilon_{1}\right) p H(t)+\varepsilon \varepsilon_{1}\|u\|_{p}^{p}
\end{aligned}
$$

From the above estimate we know there exists a constant $\gamma>0$ such that

$$
L^{\prime}(t) \geq \varepsilon \gamma\left(\left\|u_{t}\right\|_{\rho+2}^{\rho+2}+H(t)+\|u\|_{p}^{p}\right) \geq 0, \quad t>0
$$

where

$$
\gamma=\min \left\{\left(\frac{1}{\rho+1}+\frac{p\left(1-\varepsilon_{1}\right)}{\rho+2}\right),\left(1-\varepsilon_{1}\right) p, \varepsilon_{1}\right\} .
$$

Since

$$
L(0)=H^{1-\sigma}(0)+\frac{\varepsilon}{\rho+1} \int_{\Omega}\left|u_{t}\right|^{\rho} u_{1} u_{0} d x+\frac{\varepsilon}{2} \int_{\Omega}\left|\nabla u_{0}\right|^{2} d x>0,
$$


combining (3.21), we have

$$
L(t) \geq L(0)>0, \quad t>0 .
$$

We now estimate the term $\int_{\Omega}\left|u_{t}\right|^{\rho} u_{t} u d x$ as follows:

$$
\left.\left|\int_{\Omega}\right| u_{t}\right|^{\rho} u_{t} u d x \mid \leq\left\|u_{t}\right\|_{\rho+2}^{\rho+1}\|u\|_{\rho+2} \leq C\left\|u_{t}\right\|_{\rho+2}^{\rho+1}\|u\|_{p}
$$

Using Young's inequality, we have

$$
\left(\left.\left|\int_{\Omega}\right| u_{t}\right|^{\rho} u_{t} u d x \mid\right)^{\frac{1}{1-\sigma}} \leq C\left\|u_{t}\right\|_{\rho+2}^{\frac{\rho+1}{1-\sigma}}\|u\|_{p}^{\frac{1}{1-\sigma}} \leq C\left(\left\|u_{t}\right\|_{\rho+2}^{\frac{\rho+1}{1-\sigma} \mu}+\|u\|_{p}^{\frac{1}{1-\sigma} \theta}\right)
$$

where $\frac{1}{\mu}+\frac{1}{\theta}=1$. By choosing

$$
\mu=\frac{(1-\sigma)(\rho+2)}{\rho+1}>1
$$

we have

$$
\frac{\theta}{1-\sigma}=\frac{\rho+2}{(1-\sigma)(\rho+2)-(\rho+1)} .
$$

By (3.10), we know

$$
\frac{\theta}{1-\sigma}<p
$$

Then, by (3.8), we have

$$
\|u\|_{p}^{\frac{\theta}{1-\sigma}}=\|u\|_{p}^{p-\left(p-\frac{\theta}{1-\sigma}\right)}=\|u\|_{p}^{p}\|u\|_{p}^{-k} \leq\|u\|_{p}^{p} C H^{-\frac{k}{p}}(t) \leq C\|u\|_{p}^{p} H^{-\frac{k}{p}}(0),
$$

where $k=p-\frac{\theta}{1-\sigma}$ is a positive constant. Now, from (3.23), we have

$$
\left(\left.\left|\int_{\Omega}\right| u_{t}\right|^{\rho} u_{t} u d x \mid\right)^{\frac{1}{1-\sigma}} \leq C\left(\left\|u_{t}\right\|_{\rho+2}^{\rho+2}+\|u\|_{p}^{p} H^{-\frac{p}{k}}(0)\right)
$$

Therefore it follows

$$
\begin{aligned}
L^{\frac{1}{1-\sigma}}(t) & =\left(H^{1-\sigma}(t)+\frac{\varepsilon}{\rho+1} \int_{\Omega}\left|u_{t}\right|^{\rho} u_{t} u d x+\frac{\varepsilon}{2} \int_{\Omega}|\nabla u(t)|^{2} d x\right)^{\frac{1}{1-\sigma}} \\
& \leq C\left(H(t)+\left.\left.\left|\int_{\Omega}\right| u_{t}\right|^{\rho} u_{t} u d x\right|^{\frac{1}{1-\sigma}}+\left.\left.\left|\int_{\Omega}\right| \nabla u(t)\right|^{2} d x\right|^{\frac{1}{1-\sigma}}\right) \\
& \leq C\left(H(t)+\left\|u_{t}\right\|_{\rho+2}^{\rho+2}+\|u\|_{p}^{p}+\|\nabla u(t)\|_{2}^{\frac{2}{1-\sigma}}\right) .
\end{aligned}
$$

From (3.5) and (3.7), we have

$$
\|\nabla u(t)\|_{2}^{\frac{2}{1-\sigma}} \leq C^{\frac{1}{1-\sigma}} \leq \frac{C^{\frac{1}{1-\sigma}}}{H(0)} H(t) .
$$


It follows from (3.24) and (3.25) that

$$
L^{\frac{1}{1-\sigma}}(t) \leq C\left(H(t)+\left\|u_{t}\right\|_{\rho+2}^{\rho+2}+\|u\|_{p}^{p}\right) .
$$

Combining (3.21) and (3.26), we arrive at

$$
L^{\prime}(t)>\frac{\varepsilon \gamma}{C} L^{\frac{1}{1-\sigma}}(t), \quad t>0 .
$$

By a simple integration of (3.27) over $(0, t)$, we obtain

$$
L^{\sigma /(1-\sigma)}(t) \geq \frac{1}{L^{-\sigma /(1-\sigma)}(0)-\varepsilon \gamma t \sigma /[C(1-\sigma)]}, \quad t>0 .
$$

This shows that $L(t)$ blows up in finite time $T_{0}$, and

$$
T_{0} \leq \frac{C(1-\sigma)}{\varepsilon \gamma \sigma L^{\sigma /(1-\sigma)}(0)} .
$$

Furthermore, we have

$$
\lim _{t \rightarrow T_{0}^{-}}\left(\left\|u_{t}\right\|_{\rho+2}^{\rho+2}+\|\nabla u\|_{2}^{2}+\|u\|_{p}^{p}\right)=\infty
$$

This leads to a contradiction with (3.5). Thus, the solution of problem (1.1) blows up in finite time.

\section{Global existence}

In this section we show that the solution of (1.1) is global if $\rho+2 \geq p$.

Theorem 4.1 Assume that (G1), (G2) hold and $2<p \leq \rho+2$. Assume further

$$
\rho+2 \leq \frac{2(n-1)}{n-2} .
$$

Then for any initial data $\left(u_{0}, u_{1}\right) \in H_{0}^{1}(\Omega) \times L^{2}(\Omega)$, the solution of problem (1.1) exists globally.

Proof We set

$$
\begin{aligned}
F(t) & =\frac{1}{\rho+2}\left\|u_{t}\right\|_{\rho+2}^{\rho+2}+\frac{1}{2}\left(1-\int_{0}^{t} g(s) d s\right)\|\nabla u(t)\|_{2}^{2}+\frac{1}{2}(g \circ \nabla u)(t)+\frac{1}{p}\|u(t)\|_{p}^{p} \\
& =E(t)+\frac{2}{p}\|u(t)\|_{p}^{p} .
\end{aligned}
$$

Differentiating $F(t)$ and using (2.5), we get

$$
F^{\prime}(t)=E^{\prime}(t)+2 \int_{\Omega}|u|^{p-2} u u_{t} d x \leq-\left\|\nabla u_{t}\right\|_{2}^{2}+2 \int_{\Omega}|u|^{p-2} u u_{t} d x .
$$

Using the Hölder inequality and Young's inequality, we obtain the estimate

$$
\left.\left|\int_{\Omega}\right| u\right|^{p-2} u u_{t} d x \mid \leq \tilde{\varepsilon}\left\|u_{t}\right\|_{p}^{p}+C(\tilde{\varepsilon})\|u\|_{p}^{p}
$$


in which $\tilde{\varepsilon}$ is a small positive constant to be chosen later, and $C(\tilde{\varepsilon})$ is a positive constant depending on $\tilde{\varepsilon}$.

Using Lemma 2.4, (4.3) and the embedding theorem, we obtain

$$
\begin{aligned}
\left.\left|\int_{\Omega}\right| u\right|^{p-2} u u_{t} d x \mid & \leq \tilde{\varepsilon}\left\|u_{t}\right\|_{p}^{2}+C_{1} \tilde{\varepsilon}\left\|u_{t}\right\|_{\rho+2}^{\rho+2}+C(\tilde{\varepsilon})\|u\|_{p}^{p} \\
& \leq \tilde{\varepsilon} B\left\|\nabla u_{t}\right\|_{2}^{2}+C_{1} \tilde{\varepsilon}\left\|u_{t}\right\|_{\rho+2}^{\rho+2}+C(\tilde{\varepsilon})\|u\|_{p}^{p} .
\end{aligned}
$$

Substituting (4.4) to (4.2), we have

$$
F^{\prime}(t) \leq-(1-\tilde{\varepsilon} B)\left\|\nabla u_{t}\right\|_{2}^{2}+C_{1} \tilde{\varepsilon}\left\|u_{t}\right\|_{\rho+2}^{\rho+2}+C(\tilde{\varepsilon})\|u\|_{p}^{p}
$$

Choosing $\tilde{\varepsilon}=\frac{1}{2 B}$ in (4.5), we arrive at

$$
F^{\prime}(t) \leq C_{2} F(t)
$$

Furthermore we obtain

$$
F(t) \leq C_{3} e^{C_{2} t} .
$$

This completes the proof of the global existence result.

\section{Competing interests}

The authors declare that they have no competing interests.

\section{Authors' contributions}

The authors declare that the study was realized in collaboration with the same responsibility. All authors read and approved the final manuscript.

\section{Acknowledgements}

The authors are highly grateful for the referees valuable suggestions which improved this work a lot. This work was supported by NNSF of China (61374089), NSF of Shanxi Province (2014011005-2), Shanxi international science and technology cooperation projects (2014081026).

\section{Publisher's Note}

Springer Nature remains neutral with regard to jurisdictional claims in published maps and institutional affiliations.

Received: 10 February 2017 Accepted: 21 April 2017 Published online: 05 May 2017

\section{References}

1. Berrimi, S, Messaoudi, SA: Existence and decay of solutions of a viscoelastic equation with a nonlinear source. Nonlinear Anal. 64, 2314-2331 (2006)

2. Cavalcanti, MM, Domingos Cavalcanti, VN, Soriano, JA: Exponential decay for the solution of semilinear viscoelastic wave equations with localized damping. Electron. J. Differ. Equ. 2002, 44 (2002)

3. Cavalcanti, MM, Oquendo, HP: Frictional versus viscoelastic damping in a semilinear wave equation. SIAM J. Control Optim. 42, 1310-1324 (2003)

4. Cavalcanti, MM, Domingos Cavalcanti, VN, Martinez, P: General decay rate estimates for viscoelastic dissipative systems. Nonlinear Anal. 68, 177-193 (2008)

5. Cavalcanti, MM, Domingos Cavalcanti, VN, Soriano, JA: Global existence and asymptotic stability for viscoelastic problems. Differ. Integral Equ. 15, 731-748 (2002)

6. Messaoudi, SA, Tatar, NE: Exponential and polynomial decay for a quasilinear viscoelastic equation. Nonlinear Anal. 68, 785-793 (2007)

7. Munoz Rivera, JE, Baretto, R: Decay rates for viscoelastic plates with memory. J. Elast. 44,61-87 (1996)

8. Wang, YJ, Wang, YF: Exponential energy decay of solutions of viscoelastic wave equations. J. Math. Anal. Appl. 347 , 18-25 (2008)

9. Messaoudi, SA: Blow up and global existence in a nonlinear viscoelastic wave equation. Math. Nachr. 260, 58-66 (2003) 
10. Messaoudi, SA: Blow up of positive-initial-energy solutions of a nonlinear viscoelastic hyperbolic equation. J. Math. Anal. Appl. 320, 902-915 (2006)

11. Lu, LQ, Li, SJ: Cauchy problem for a nonlinear viscoelastic equation with nonlinear damping and source terms. Appl. Math. Lett. 24, 1275-1281 (2011)

12. Guo, L, Yuan, ZQ, Lin, GG: Blow up and global existence for a nonlinear viscoelastic wave equation with strong damping and nonlinear damping and source terms. Appl. Math. Lett. 6, 806-816 (2015)

13. Song, HT, Zhong, CK: Blow up of solutions of a nonlinear viscoelastic wave equation. Nonlinear Anal., Real World Appl. 11, 3877-3883 (2010)

14. Payne, L, Sattinger, D: Saddle points and instability on nonlinear hyperbolic equations. Isr. J. Math. 22, 273-303 (1975)

15. Song, HT, Xue, DS: Blow up of solutions for a viscoelastic wave equation with strong damping. Nonlinear Anal. 109, 245-251 (2014)

16. Cavalcanti, MM, Domingos Cavalcanti, VN, Ferreira, J: Existence and uniform decay for nonlinear viscoelastic equation with strong damping. Math. Methods Appl. Sci. 24, 1043-1053 (2001)

17. Cavalcanti, MM, Domingos Cavalcanti, VN, Lasiecka, I, Webler, CM: Intrinsic decay rates for the energy of a nonlinear viscoelastic equation modeling the vibrations of thin rods with variable density. Adv. Nonlinear Anal. doi:10.1515/anona-2016-0027

18. Liu, WJ: General decay and blow-up of solution for a quasilinear viscoelastic problem with nonlinear source Nonlinear Anal. 73, 1890-1904 (2010)

19. Song, HT: Global nonexistence of positive initial energy solutions for a viscoelastic wave equation. Nonlinear Anal. $125,260-269$ (2015)

\section{Submit your manuscript to a SpringerOpen ${ }^{\circ}$ journal and benefit from:}

- Convenient online submission

- Rigorous peer review

Immediate publication on acceptance

- Open access: articles freely available online

- High visibility within the field

- Retaining the copyright to your article 